\title{
A Consensus Statement on the Sleep Medicine Practice during the COVID-19 Pandemic
}

Jagdish C Suri ${ }^{1}$, Nagarajan Ramakrishnan ${ }^{2}$, AG Ghoshal $^{3}$, Dipak Bhattacharya ${ }^{4}$, Dipti Gothi ${ }^{5}$, Dipankar Dutta ${ }^{6}$, Gopal Raval $^{7}$, Manoj Goel ${ }^{8}$, RMPL Ramanathan ${ }^{9}$, Seemab Shaikh ${ }^{10}$, Sushant Khurana ${ }^{11}$, Tejas M Suri ${ }^{12}$

\begin{abstract}
The coronavirus disease 2019 (COVID-19) pandemic has become a major public health concern worldwide profoundly impacting various aspects of the practice of sleep medicine. Nonetheless, new onset sleep disturbances including insomnia and circadian rhythm disorders have been reported during this period. Severe COVID-19 disease and obstructive sleep apnea (OSA) may have shared risk factors including advancing age, systemic hypertension, and cardiovascular disease. In light of the unprecedented situation and the lack of definitive evidence on ways to tackle the same, sleep physicians and surgeons from the Indian Sleep Disorders Association have prepared this consensus statement on the practice of sleep medicine in India during the COVID-19 pandemic. This document carries expert recommendations under the following categories: (i) sleep clinics and telemedicine, (ii) home sleep apnea testing (HSAT), (iii) in-laboratory polysomnography (PSG) and positive airway pressure (PAP) titration, and (iv) use of PAP devices in OSA. We hope that it will serve as a reference guide to sleep physicians to restart their practice in a safe manner during the COVID-19 pandemic.

Keywords: COVID-19, Guidelines, Obstructive sleep apnea, Sleep practice, Sleep studies.

Indian Journal of Sleep Medicine (2020): 10.5005/jp-journals-10069-0051
\end{abstract}

\section{Executive Summary}

\section{Sleep Clinic and Telemedicine}

- Telemedicine consults may be preferable for follow-up consults of existing patients with sleep disorders. The use of remote monitoring (e.g., compliance with positive airway pressure (PAP) therapy) may be considered where feasible.

- Telemedicine consults may be considered for selected consults of new patients (e.g., lockdown sleep syndrome).

- Routine in-person clinic visits should be postponed where feasible. However, in-person visits may be conducted for selected patients with suspected sleep-disordered breathing with urgent indications for testing and treatment.

- If in-person clinic visits are conducted, infection prevention and control measures should be observed including staggered appointment scheduling, physical distancing, use of masks, and hand hygiene. Patients and staff members should be screened at entry points for fever or respiratory symptoms.

\section{Home Sleep Apnea Testing}

- Home sleep apnea testing (HSAT) may be considered only in patients with suspected obstructive sleep apnea (OSA) with high pretest probability and in absence of usual contraindications.

- If HSAT is performed, test equipment can be remotely delivered to the patient (e.g., doorstep or courier delivery) along with user instructions via pamphlet, video, or telemedicine.

- Single-use sensors and cannulas should not be reused.

- The test equipment must be disinfected using manufacturer's recommendations and should not be reused for at least 72 hours.

\section{In-laboratory Polysomnography and Positive Airway Pressure Titration}

- Routine in-laboratory polysomnography (PSG) should be postponed where feasible. However, in-person laboratory
${ }^{1} \mathrm{JCS}$ Institute of Pulmonary, Critical Care and Sleep Medicine/Fortis Flt. Lt. Rajan Dhall Hospital, New Delhi, India

${ }^{2}$ Nithra Institute of Sleep Sciences, Chennai, Tamil Nadu, India

${ }^{3}$ National Allergy Asthma Bronchitis Institute, Kolkata, West Bengal, India

${ }^{4}$ Department of Pulmonary, Critical Care and Sleep Medicine, Vardhman Mahavir Medical College and Safdarjung Hospital, New Delhi, India

${ }^{5}$ Department of Pulmonary, Sleep and Critical Care Medicine, ESIPGIMSR, New Delhi, India

${ }^{6}$ Department of ENT and Sleep Disorders, Belle Vue Clinic, Kolkata,West Bengal, India

${ }^{7}$ Department of Pulmonary and Critical Care, SPARSH Chest Disease Centre, Ahmedabad/HCG Multispecialty Hospital, Ahmedabad/ Sterling Hospital, Ahmedabad, Gujarat, India

${ }^{8}$ Department of Pulmonology and Critical Care, Fortis Memorial Research Institute, Gurugram, Haryana, India

${ }^{9} \mathrm{KMCH}$ Center for Advanced Lung Diseases and Transplantation, Coimbatore, Tamil Nadu, India

${ }^{10}$ Inamdar Multispeciality Hospital, Pune/KEM Hospital, Pune/Sahyadri Group of Hospitals, Pune, Maharashtra, India

${ }^{11}$ Sleep and Respiratory Care, Philips India Ltd., Gurugram, Haryana, India

${ }^{12}$ Department of Pulmonary, Critical Care and Sleep Medicine, All India Institute of Medical Sciences, New Delhi, India

Corresponding Author: Jagdish C Suri, JCS Institute of Pulmonary Critical Care and Sleep Medicine/Fortis Flt. Lt. Rajan Dhall Hospital, New Delhi, India, e-mail: docjcsuri@gmail.com

How to cite this article: Suri JC, Ramakrishnan N, Ghoshal AG, et al. A Consensus Statement on the Sleep Medicine Practice during the COVID-19 Pandemic. Indian J Sleep Med 2020;15(2):21-25.

Source of support: Nil

Conflict of interest: None

() The Author(s). 2020 Open Access This article is distributed under the terms of the Creative Commons Attribution 4.0 International License (https://creativecommons. org/licenses/by-nc/4.0/), which permits unrestricted use, distribution, and non-commercial reproduction in any medium, provided you give appropriate credit to the original author(s) and the source, provide a link to the Creative Commons license, and indicate if changes were made. The Creative Commons Public Domain Dedication waiver (http://creativecommons.org/publicdomain/zero/1.0/) applies to the data made available in this article, unless otherwise stated. 
testing may be conducted for selected patients with suspected sleep-disordered breathing with urgent indications for testing and treatment and contraindications for HSAT.

- If in-laboratory PSG is performed, it is advisable to screen the patient using the history of acute respiratory illness in the appropriate time frame. The decision to perform COVID-19 rapid antigen testing in asymptomatic patients prior to PSG may be individualized depending on whether PAP titration is being performed, and availability and cost implications of the test.

- In-laboratory PSG should be postponed in patients with symptomatic acute respiratory illness or positive COVID-19 microbiological test.

- If in-laboratory PSG is performed, the sleep technician should use appropriate personal protective equipment. During PAP titration, the technician wear an N-95 respirator, eye protection, gloves, and gown.

- In-laboratory PSG should preferably be performed in an airborne infection isolation room or a laboratory with placement of HEPA filter system.

\section{Use of Positive Airway Pressure Devices in Obstructive Sleep Apnea}

- Positive airway pressure users with preexisting OSA should continue using their PAP devices if they have no acute respiratory symptoms or history of contact with a COVID-19 patient.

- Positive airway pressure users should prefer the use of oronasal mask in favor of other interfaces and avoid use of humidifiers.

- All PAP users should be advised to routinely clean their PAP devices as per the manufacturer's instructions.

- Positive airway pressure users with preexisting OSA who develop suspected/confirmed mild COVID-19 managed using home isolation may consider continuing PAP devices with strict isolation precautions.

- Positive airway pressure users with preexisting OSA who develop suspected/confirmed moderate or severe COVID-19 requiring hospital admission should be managed as per the discretion of the treating physician.

- New PAP users may be educated regarding PAP usage through pamphlets or video. If in-person training is necessary, then the sleep technician must use personal protective equipment including N-95 respirator, eye protection, gloves, and gown.

- Patients with OSA who are unable to use PAP devices must be advised to avoid sleep in supine position, to abstain from alcohol/sedatives, to use nasal decongestants as indicated, and to continue lifestyle modifications including weight loss.

\section{INTRODUCTION}

The coronavirus disease 2019 (COVID-19), which emerged in late 2019 in China, has rapidly engulfed the entire world, leading to it being declared a pandemic. Millions of people have been affected worldwide with India accounting for the third-highest number of cases (approximately 850,000 ) as on July $12,2020 .{ }^{1}$ The causative agent, the severe acute respiratory syndrome coronavirus-2 (SARS-CoV-2), spreads primarily via the respiratory droplet route, with a probable contribution of aerosol-related transmission. Furthermore, the virus has been found to survive for hours to a few days on external surfaces, such as wood, plastic, and stainless steel; raising concerns about fomite transmission. ${ }^{2}$ Transmission to both patients and healthcare workers in the healthcare setting has been reported. ${ }^{3}$ The disease has substantial morbidity and mortality, which led to extreme public health interventions including lockdowns and temporary suspension of non-emergent healthcare services in many parts of the world.

The COVID-19 pandemic has profoundly impacted various aspects of the practice of sleep medicine. The conduct of sleep clinics and sleep testing has been interrupted worldwide due to the aforementioned public health measures; only $20 \%$ of sleep centers were providing services at the peak of the pandemic in Europe. ${ }^{4}$ It is notable that the risk factors for obstructive sleep apnea (OSA), such as advancing age, obesity, hypertension, diabetes mellitus, and cardiovascular disease, also portend a greater risk for severe and life-threatening COVID-19. ${ }^{5}$ This suggests further exploration of the role of OSA in worsening hypoxemia in COVID-19, and conversely merits study of the protective impact of positive airway pressure (PAP) therapy. ${ }^{6}$ Unfortunately, both PAP titration in-laboratory and use of PAP devices at home for managing OSA are associated with concerns of aerosolization, thereby invoking infection control implications. ${ }^{7}$ Besides OSA, there is increased incidence of newonset sleep problems and worsening of preexisting problems including insomnia and delayed sleep phase syndrome as the majority of the population remains locked in their homes. ${ }^{8}$

These challenges have required a relook into the practice of sleep medicine in the context of the pandemic. The use of technologies, such as telemedicine and remote monitoring, have generated a renewed interest. Simultaneously, there is increasing emphasis on triage and infection prevention and control (IPC) for in-person clinic visits and inpatient laboratory services. In light of this unprecedented situation and the lack of definitive evidence on ways to tackle the same, a diverse group of experts representing the Indian Sleep Disorders Association (ISDA) have framed this consensus statement on the practice of sleep medicine in India during the COVID-19 pandemic.

\section{Materials and Methods}

A group of experienced sleep physicians including pulmonologists, internists, and otorhinolaryngologists from different parts of India were contacted using email and asked to identify key areas of discussion. The group had representations from public and private sector physicians practising in both in-hospital and standalone sleep centers. The group identified the following four areas of discussion in context of the COVID19 pandemic: the conduct of sleep clinics (including use of telemedicine), home sleep apnea testing (HSAT), in-laboratory polysomnography (PSG), and the management of OSA patients using PAP devices. The group subsequently convened to deliberate on these issues via video conferencing. In the absence of any definitive evidence to guide decisions, the group framed recommendations based on consensus, keeping in mind the policies and regulations of the national health authorities. There was no attempt to grade these recommendations as they were based on expert opinion. The group recognizes that our understanding of COVID-19 and public health responses to the same are rapidly evolving and hence these recommendations may be supplanted as new evidence emerges. Hence, readers are advised to remain updated with the directions of the local governmental health authorities.

\section{Sleep Clinics and Telemedicine}

The use of travel restrictions and lockdown in parts of the world combined with minimization of non-emergent healthcare services in 
favor of emergency and pandemic services has led to reduced patient access to sleep services. Patients suffering from sleep disorders are unable to come for follow-up visits, which may hamper their treatment. Furthermore, new onset sleep problems have been noted among the populace stuck at home during this period. The stresses associated with fear of the disease, job loss, uncertainty regarding the future, and social isolation have contributed to occurrence of insomnia. ${ }^{8}$ One of the authors has described this in an unpublished report as "lockdown sleep syndrome" and the expert panel agreed to apply this terminology (Ramakrishnan N 2020, personal communication, July 12). Furthermore, people who work from home may suffer from an alteration of the sleep wake cycle (e.g., delayed sleep phase syndrome). ${ }^{9}$ The use of caffeinated and alcoholic beverages for coping further exacerbates the situation. Furthermore, home isolation may increase sedentary lifestyles and poor dietary habits contributing to new or worsening OSA. These considerations raise alarm as sleep deprivation is associated with immune disturbances and has been found to increase the risk of viral respiratory illness. ${ }^{10}$ Furthermore, untreated OSA may lead to nocturnal hypoxemia which may theoretically worsen outcomes in COVID-19. ${ }^{6}$

Hence, it is essential that sleep services are made available for both new and existing patients. This is fraught with considerable challenges given the risk of healthcare-related infection transmission. Physical distancing norms necessitates that fewer staff attend clinic and the rest work from home. Furthermore, reduced in-person patient attendance to the sleep clinic by prioritizing the most urgent visits only is imperative. In such a situation, the delivery of sleep clinic services can be achieved with the help of adoption of telemedicine technologies including video or audio consults. The recently released Indian Telemedicine Practice Guidelines provide practical advice to doctors for enabling rational teleconsultation (accessible on https://www.mohfw.gov.in/pdf/Telemedicine. pdf). ${ }^{11}$ Telemedicine is well suited for follow-up consults wherein patient records are available. Positive airway pressure usage data acquired by the healthcare provider using remote monitoring can help improve PAP compliance. ${ }^{12}$ Furthermore, counseling and prescriptions for "lockdown sleep syndrome" can be provided using telemedicine, while following the framework of the Telemedicine Practice Guidelines.

For patients with suspected new onset sleep-disordered breathing, in-person evaluation may be necessary for proper comorbidity assessment, anthropometric, and physical examination. In such situations, priority should be given to those patients with need for urgent testing and treatment (respiratory failure, right heart failure, congestive heart failure or excessive sleepiness with risk of work-related accidents, and motor vehicular accidents). The steps to be taken for infection prevention in the sleep clinic include screening of patients and staff for acute respiratory symptoms and fever at entry points; mandatory mask usage by patients and staff; hand hygiene with alcohol-based hand rub or soap; and physical distancing measures (chairs in waiting room 6 feet apart, removing communal items like magazines, disallowing non-essential patient attendants, and staggered appointment scheduling). Educational posters regarding precautions against COVID-19 may be displayed at prominent places inside the clinic. ${ }^{13}$

\section{Key Recommendations}

- Telemedicine consults may be preferable for follow-up consults of existing patients with sleep disorders. The use of remote monitoring (e.g., compliance with PAP therapy) may be considered where feasible.
- Telemedicine consults may be considered for selected consults of new patients (e.g., lockdown sleep syndrome).

- Routine in-person clinic visits should be postponed where feasible. However, in-person visits may be conducted for selected patients with suspected sleep-disordered breathing with urgent indications for testing and treatment.

- If in-person clinic visits are conducted, IPC measures should be observed including staggered appointment scheduling, physical distancing, use of masks, and hand hygiene. Patients and staff members should be screened at entry points for fever or respiratory symptoms.

\section{Home Sleep Apnea Testing}

Sleep apnea testing at home using level 3 portable sleep tests has been found to be useful in patients with high pretest probability of OSA. Home Sleep Apnea Testing can be combined with therapy using an autotitrating PAP device at home, thus obviating need for in-laboratory manual titration. ${ }^{14}$ However, HSAT may often produce indeterminate or false-negative results; the latter is often observed in mild OSA. In such situations, a follow-up in-laboratory PSG is required. ${ }^{15}$ Furthermore, an in-laboratory study and titration is imperative in situations where complex patterns of sleep-disordered breathing is expected (e.g., hypoventilation syndromes and central sleep apnea). Hence, HSAT is currently not recommended in patients with comorbidities including heart failure, chronic respiratory disease, stroke, neuromuscular disorders, obesity hypoventilation syndrome, and opioid use disorder. ${ }^{14}$ These patient populations should not be offered HSAT during the pandemic period due to uncertain efficacy and safety of such a management strategy.

Hence, HSAT may obviate the need for in-laboratory PSG in a carefully selected patient population. However, risk of infection transmission during HSAT to patients and sleep technicians still exists. This can be overcome using a strategy of remote delivery of the portable testing equipment to the patient, using doorstep or courier delivery without face-to-face contact. Hence, the technician can instruct the patient on the use of the test device via preprepared pamphlets/videos or by telemedicine (audio or video calls). Singleuse sensors and cannulas should not be reused. Furthermore, after the device is returned, it should be disinfected using the manufacturer's guidelines and should not be reused for at least 72 hours. The latter recommendation stems from the observation that SARS-CoV-2 survives on plastic for up to 72 hours. $^{2}$

\section{Key Recommendations}

- Home sleep apnea testing may be considered only in patients with suspected OSA with high pretest probability and in absence of usual contraindications.

- If HSAT is performed, test equipment can be remotely delivered to the patient (e.g., doorstep or courier delivery) along with user instructions via pamphlet, video, or telemedicine.

- Single-use sensors and cannulas should not be reused.

- The test equipment must be disinfected using the manufacturer's recommendations and should not be reused for at least 72 hours.

\section{In-laboratory Polysomnography and Positive Airway Pressure Titration}

In the previous section, we highlighted that patients with comorbidities, such as chronic respiratory disease, heart failure, stroke, neuromuscular disease, obesity hypoventilation syndrome, and chronic opioid use, require in-laboratory PSG and titration. During the pandemic period, it is essential to triage such patients 
with suspected OSA to ensure that in-laboratory testing is prioritized to those with urgent indications for testing and treatment (e.g., congestive heart failure, right heart failure, nocturnal angina, or respiratory failure). ${ }^{16}$ Furthermore, patients with excessive daytime sleepiness in critical professions (e.g., airline pilots, long distance truck drivers, etc.) who are ineligible for HSAT require urgent sleep apnea testing and treatment. All routine in-laboratory PSGs may be postponed during the pandemic.

All patients who are planned for in-lab PSG should be screened telephonically prior to arrival to the laboratory for any new acute respiratory symptoms or fever. Symptomatic patients should be referred for COVID-19 RT-PCR testing through the appropriate pathway and their sleep study should be deferred. The Indian Council of Medical Research has recently recommended the use of COVID-19 Standard Q rapid antigen test (SD Biosensor) for all asymptomatic patients planned for aerosol-generating procedures. ${ }^{17}$ In-lab PAP titration is a potentially aerosol-generating procedure. In cognizance of the above, COVID-19 rapid antigen testing may be considered in asymptomatic patients undergoing in-laboratory PSG and PAP titration keeping in mind the availability and cost implications. The expert group recommends individualized decision by the clinician in such situations.

The sleep technician should be appropriately trained in droplet and aerosol precautions prior to initiation of in-laboratory sleep testing. The PSG should be ideally conducted in an airborne infection isolation room (negative pressure room) or a laboratory with placement of HEPA filter system. The technician's station should be in a separate room with remote monitoring ability. The technician should wear an N-95 respirator, eye protection (goggles or face shield), gloves, and gown while entering the patient's room during PAP titration. ${ }^{18}$ Disinfection of testing equipment between studies should be performed using the manufacturer's guidelines.

\section{Key Recommendations}

- Routine in-laboratory PSG should be postponed where feasible. However, in-person laboratory testing may be conducted for selected patients with suspected sleep-disordered breathing with urgent indications for testing and treatment and contraindications for HSAT.

- If in-laboratory PSG is to be performed, it is advisable to screen the patient using the history of acute respiratory illness in the appropriate period. The decision to perform COVID-19 rapid antigen testing in asymptomatic patients prior to PSG may be individualized depending on whether PAP titration is being performed, and availability and cost implications of the test.

- In-laboratory PSG should be postponed in patients with symptomatic acute respiratory illness or positive COVID-19 microbiological test.

- If in-laboratory PSG is performed, the sleep technician should use appropriate personal protective equipment. During PAP titration, the technician wear an $\mathrm{N}-95$ respirator, eye protection, gloves, and gown.

- In-laboratory PSG should preferably be performed in an airborne infection isolation room or a laboratory with placement of HEPA filter system.

\section{Use of Positive Airway Pressure Devices in Obstructive Sleep Apnea}

The use of PAP devices has been found to generate aerosol, thereby raising concerns regarding transmission of infection to family members and caregivers. In a study of 86 hospital wards in Hong Kong during the SARS epidemic, it was found that wards with use of bilevel PAP ventilation had an 11-fold increased risk of superspreading events. ${ }^{19}$ In another study by Hui et al., a human patient simulator was used to assess exhaled air dispersion during use of continuous positive airway pressure (CPAP) and highflow nasal cannula (HFNC). ${ }^{20}$ The authors found that exhaled air dispersion is least during CPAP using oronasal mask compared with CPAP using nasal pillows or HFNC. Furthermore, the dispersion increases as the pressure is increased or fitting is loosened.

Despite these concerns, the discontinuation of PAP therapy for an indefinite duration encompassing the ongoing pandemic is impractical. Although PAP for management of OSA may not be considered as a life-sustaining measure, prolonged discontinuation is likely to cause increase in symptoms (daytime sleepiness, impaired concentration, mood disturbances) and increase the risk of comorbidities and accidents. ${ }^{21}$ Uncontrolled OSA may have cardiovascular implications which may potentially lead to adverse outcomes. ${ }^{5}$ Hence, the group came to a consensus that existing PAP users with preexisting OSA should continue to use their devices if they do not have acute respiratory symptoms or contact with a COVID-19 patient. Furthermore, the use of oronasal mask interface may be preferred over nasal mask or nasal pillow. Adequate fit should be ensured to minimize leak. Since PAP use at home employs a vented mask, there is release of unfiltered exhaled air in the environment. To overcome this, the use of a non-vented full-face mask with inline heat moisture exchanger (HME) or viral filter and a whisper swivel expiration port has been described. This allows exhaled air to pass through a viral filter $199.999 \%$ filtration) prior to exit into the environment. ${ }^{22,23}$ Other approaches to prevent aerosolization include avoidance of humidifier use, and regular cleaning of PAP devices as per the manufacturer's recommendations.

Among PAP users with preexisting OSA who develop suspected or confirmed mild COVID-19, the use of PAP therapy must be approached with caution. If the patient is eligible for home therapy, they must observe strict isolation in a separate room from remaining household members. In such a situation, it may be feasible to continue PAP therapy as long as isolation is maintained. However, the decision should be taken in consultation with the sleep physician considering the benefits to the patients and risk to the domestic contacts. The caregivers must be educated regarding aerosol precautions to be observed while entering the patient's room as the virus may remain viable in aerosol for up to 3 hours. $^{2}$

If the PAP user with preexisting OSA develops moderate or severe suspected or confirmed COVID-19, they would usually require hospital admission as per the current Indian guidelines. Such patients may often require supplemental oxygen, noninvasive ventilation, high-flow nasal oxygen, or mechanical ventilation. The decision to use PAP therapy or a ventilatory strategy should be made by the treating physician depending on the clinical context. The patient may be advised to bring their PAP device to the hospital. ${ }^{24}$ Although the use of a critical care ventilator with non-vented mask for PAP therapy in-hospital may be preferable to reduce aerosolization, there is a need to prioritize ventilator usage for managing respiratory failure in a pandemic scenario with potential shortages.

Patients with newly diagnosed OSA and initiated on PAP therapy need to be adequately educated about and familiarized 
with their PAP devices. Patient experience with PAP devices during initiation of therapy may determine long-term compliance to therapy. ${ }^{25}$ Although the optimal approach to PAP education during the pandemic needs to be further explored, the group concurred that the use of pamphlets and videos for the same should be encouraged. If in-person training in the laboratory is necessary, the technician should observe aerosol precautions and use personal protective equipment accordingly ( $\mathrm{N}-95$ respirator, eye protection, gloves, and gown). Such PAP training must be performed in an airborne infection isolation room.

All patients with OSA should be encouraged to continue lifestyle modification including regular physical exercise (while observing physical distancing norms) and weight loss. Furthermore, those patients who are unable to use their PAP devices should be advised regarding avoidance of supine position during sleep, abstinence from alcohol and sedatives, and use of nasal decongestants as indicated. ${ }^{16}$

\section{Key Recommendations}

- Positive airway pressure users with preexisting OSA should continue using their PAP devices if they have no acute respiratory symptoms or history of contact with a COVID-19 patient.

- Positive airway pressure users should prefer the use of oronasal mask in favor of other interfaces and avoid use of humidifiers.

- All PAP users should be advised to routinely clean their PAP devices as per the manufacturer's instructions.

- Positive airway pressure users with preexisting OSA who develop suspected/confirmed mild COVID-19 managed using home isolation may consider continuing PAP devices with strict isolation precautions.

- Positive airway pressure users with preexisting OSA who develop suspected/confirmed moderate or severe COVID-19 requiring hospital admission should be managed as per the discretion of the treating physician.

- New PAP users may be educated regarding PAP usage through pamphlets or video. If in-person training is necessary, then the sleep technician must use personal protective equipment including N-95 respirator, eye protection, gloves, and gown.

- Patients with OSA who are unable to use PAP devices must be advised to avoid sleep in supine position, to abstain from alcohol/sedatives, to use nasal decongestants as indicated, and to continue lifestyle modifications including weight loss.

\section{References}

1. WHO Coronavirus Disease (COVID-19) Dashboard [Internet]. [cited 2020 Jul 5]. Available from: https://covid19.who.int.

2. van Doremalen N, Bushmaker T, Morris DH, et al. Aerosol and surface stability of SARS-CoV-2 as compared with SARS-CoV-1. N Engl J Med 2020;382(16):1564-1567. DOI: 10.1056/NEJMc2004973.

3. Heinzerling A, Stuckey MJ, Scheuer T, et al. Transmission of COVID19 to health care personnel during exposures to a hospitalized patient - Solano county, California, february 2020. MMWR Morb Mortal Wkly Rep 2020;69(15):472-476. DOI: 10.15585/mmwr. $\mathrm{mm} 6915 \mathrm{e}$.

4. Grote L, McNicholas WT, Hedner J. Sleep apnoea management in Europe during the COVID-19 pandemic: data from the european sleep apnoea database (ESADA). Eur Respir J 2020;55(6):2001323. DOI: 10.1183/13993003.01323-2020.

5. McSharry D, Malhotra A. Potential influences of obstructive sleep apnea and obesity on COVID-19 severity. J Clin Sleep Med [Internet] 2020. Available from: http://jcsm.aasm.org/doi/10.5664/ jcsm. 8538 .
6. Pazarlı AC, Ekiz T, illik F. Coronavirus disease 2019 and obstructive sleep apnea syndrome. Sleep Breath [Internet] 2020. Available from: http://link.springer.com/10.1007/s11325-020-02087-0.

7. Barker J, Oyefeso O, Koeckerling D, et al. COVID-19: community CPAP and NIV should be stopped unless medically necessary to support life. Thorax 2020;75(5):367. DOI: 10.1136/thoraxjnl-2020214890.

8. Kokou-Kpolou CK, Megalakaki O, Laimou D, et al. Insomnia during COVID-19 pandemic and lockdown: prevalence, severity, and associated risk factors in French population. Psychiatry Res 2020;290:113128. DOI: 10.1016/j.psychres.2020.113128.

9. Javaheri S, Javaheri S. The impact of SARS-CoV-2 on sleep. J Clin Sleep Med [Internet] 2020. Available from: http://jcsm.aasm.org/ doi/10.5664/jcsm.8598.

10. Prather AA, Janicki-Deverts D, Hall MH, et al. Behaviorally Assessed sleep and susceptibility to the common cold. Sleep 2015;38(9):13531359. DOI: $10.5665 /$ sleep.4968.

11. Telemedicine Practice Guidelines [Internet]. 2020 [cited 2020 Jul 5]. Available from: https://www.mohfw.gov.in/pdf/Telemedicine.pdf.

12. Clavaud F, Cooper V. Does CPAP remote monitoring improves compliance and adherence? Eur Respir J [Internet] 2016;48(Suppl 60). Available from: https://erj.ersjournals.com/content/48/suppl_60/ PA3428.

13. COVID-19 mitigation strategies for sleep clinics and sleep centers - REOPENING [Internet]. Available from: https://aasm.org/covid-19resources/covid-19-mitigation-strategies-sleep-clinics-labs/.

14. Collop NA, Anderson WM, Boehlecke B, et al. Clinical guidelines for the use of unattended portable monitors in the diagnosis of obstructive sleep apnea in adult patients. Portable monitoring task force of the american academy of sleep medicine. J Clin Sleep Med JCSM Off Publ Am Acad Sleep Med 2007;3(7):737-747.

15. Kapur VK, Auckley DH, Chowdhuri S, et al. Clinical practice guideline for diagnostic testing for adult obstructive sleep apnea: an american academy of sleep medicine clinical practice guideline. J Clin Sleep Med JCSM Off Publ Am Acad Sleep Med 2017;13(3):479-504. DOI: 10.5664/jcsm.6506.

16. Ayas NT, Fraser KL, Giannouli E, et al. Helping canadian health care providers to optimize sleep disordered breathing management for their patients during the COVID-19 pandemic. Can J Respir Crit Care Sleep Med 2020;4(2):81-82.

17. Newer Additional Strategies for COVID-19 Testing [Internet]. Indian Council of Medical Research, New Delhi. 2020 [cited 2020 Jul 6]. Available from: https://www.icmr.gov.in/pdf/covid/strategy/New_ additional_Advisory_23062020_2.pdf.

18. Interim infection prevention and control recommendations for healthcare personnel during the coronavirus disease 2019 (COVID-19) pandemic. Cent Disease Cont Prevent 2020.

19. Yu IT, Xie ZH, Tsoi KK, et al. Why did outbreaks of severe acute respiratory syndrome occur in some hospital wards but not in Others? Clin Infect Dis 2007;44(8):1017-1025. DOI: 10.1086/512819.

20. Hui DS, Chow BK, Lo T, et al. Exhaled air dispersion during high-flow nasal cannula therapy versus CPAP via different masks. Eur Respir J 2019;53(4):1802339. DOI: 10.1183/13993003.02339-2018.

21. Baker JG, Sovani M. Case for continuing community NIV and CPAP during the COVID-19 epidemic. Thorax 2020;75(5):368-368. DOI: 10.1136/thoraxjnl-2020-214913.

22. Kryger M, Thomas R. Home PAP devices in COVID-19 infected patients. J Clin Sleep Med [Internet] 2020(7). Available from: http://jcsm.aasm. org/doi/10.5664/jcsm.8490.

23. Drummond M. Sleep labs, lung function tests and COVID-19 pandemic - only emergencies allowed!. Pulmonol 2020(4):S2531043720300891. DOI: 10.1016/j.pulmoe.2020.04.002.

24. Lance CG. PAP therapy increases the risk of transmission of COVID-19. Cleve Clin J Med 2020. DOI: 10.3949/ccjm.87a.ccc003ccjom;ccjm.87a. ccc003v2.

25. Lewis KE, Seale L, Bartle IE, et al. Early predictors of CPAP use for the treatment of obstructive sleep apnea. Sleep 2004;27(1):134-138. DOI: 10.1093/sleep/27.1.134. 